\title{
Hyperbaric Oxygen Therapy for Central Retinal Artery Occlusion: Patient Selection and Perspectives
}

\author{
Ali Riza Cenk Celebi \\ Atakent Education and Research \\ Hospital, Department of Ophthalmology, \\ Acibadem University School of Medicine, \\ Istanbul, Turkey
}

\begin{abstract}
The central retinal artery occlusion (CRAO) is a rare ophthalmological emergency that can occur in the eye. CRAO can affect persons of any age, however it is most common in people over the age of 60 . CRAO is associated with a number of risk factors, including giant cell arteritis, carotid artery atherosclerosis, cardiogenic emboli, hypertension, smoking, diabetes, and thromboembolic disease. The chance of each of these etiologies being present is assessed during the course of the investigation. Hyperbaric oxygen treatment (HBOT) is classified by the American Heart Association for CRAO at level IIb. In accordance with that, HBOT might be considered for the treatment of such a severe condition. HBOT can maintain retinal oxygenation during ischemic events by allowing oxygen to diffuse through choroidal capillaries that have been exposed to elevated partial pressures of oxygen. As a result, ischemia-related damage is reversed if applied within proper time frame. The amount of time that has passed prior to initiation of HBOT is considered to be the most critical factor in determining the best visual prognosis. According to the Undersea and Hyperbaric Medical Society, patients who are identified with CRAO after the onset of symptoms should be evaluated for HBOT within 24 hours. HBOT has the advantage of having a low risk profile, and it can be utilized to improve visual outcomes in proper patients.
\end{abstract}

Keywords: hyperbaric oxygen therapy, HBOT, central retinal artery occlusion, CRAO

\section{Introduction}

Central retinal artery occlusion (CRAO), which is the visual counterpart of a cerebral stroke, is considered an ophthalmological emergency. It is estimated that one in every 100,000 patients will be affected on an annual basis. ${ }^{1}$ Patients frequently present with a fast, monocular, and painless visual loss that results in visual acuity (VA) of $20 / 400$ or worse in $80 \%$ of cases if the condition is not treated. ${ }^{2}$ Patients suffering from this ailment commonly have vision loss ranging from finger counting to light perception, among other symptoms. When the flow of blood to the central retinal artery is interrupted, it results in ischemia and infarction of the retina, which is referred to as central retinal artery occlusion (CRAO). In addition, the retina consumes oxygen at a high rate $(13 \mathrm{~mL} / 100 \mathrm{~g} / \text { minute })^{3}$ and is thus prone to ischemia as a result of an intolerance to protracted oxygen deprivation as a result of its high oxygen consumption rate.

The retinal oxygen deprivation in animal models causes irreversible tissue damage after 97 minutes, and occlusions lasting more than 100 minutes cause
Correspondence: Ali Riza Cenk Celebi Atakent Education and Research Hospital, Department of Ophthalmology, Acibadem University School of Medicine, Istanbul, 34303, Turkey

Tel +902I24044084

Email arcenkcelebi@gmail.com; cenk. celebi@acibadem.edu.tr 
varying degrees of retinal damage, with significant damage happening after 240 minutes. ${ }^{4}$ It is usually severe and persistent when blood supply to the retina is interrupted for more than 4 hours, resulting in permanent retinal damage and visual loss. ${ }^{5}$ It is unclear when irreversible anoxic retinal damage occurs in humans, in contrast to animal models (some authors propose an elapsed time of approximately 6-6.5 hours, however this time frame is not trustworthy because to the wide diversity between patients, types of occlusions, and residual perfusion) ${ }^{6}$

\section{Review of the Anatomy of the CRA in Relation to the CRAO}

In addition to providing oxygen and nutrition to the eyes, the ophthalmic artery, which is a branch of the cavernous section of the internal carotid artery, is also responsible for sending blood to the brain. With the optic nerve, the central retinal artery enters the globe and distributes blood to the inner layers of the retina by its multiple branches. It is a minor branch of the ophthalmic artery and an important little branch of that artery, and it enters the globe with the optic nerve. ${ }^{7}$ If the amount of occlusion is at the level of the ophthalmic artery, it is probable that the patient will have no treatment alternatives open to them. Due to a blockage in the posterior ciliary circulation, no collateral circulation can reach the inner layers of the retinal pigment epithelium, resulting in the development of this devastating situation. ${ }^{8}$ The retina receives a two-way flow of oxygen and nutrients, with the central retinal artery sending blood to the inner layers and the choroidal circulation supplying blood to the outer layers of the retina. Only a few blood arteries are responsible for providing the sole food for the retinal tissue that is more significant than the central retinal artery, which has an average diameter of $0.16 \mathrm{~mm} .{ }^{9}$ On a cellular basis; cells in the layers of the retina whose nuclei are located within the vascularized inner retina (such as ganglion cells, bipolar cells, and Müller glial cells) receive their oxygen from the central retinal artery, whereas those cells in the layers of the retina whose nuclei are located within the capillary-free outer retina (such as photoreceptors and retinal pigment epithelium) receive their oxygen from the adjacent choroid, which is a component of the posterior ciliary arterial circulation. ${ }^{10}$

Acute pan-retinal hypoperfusion results in ischemia in the inner retinal layers (ganglion cell layer and inner nuclear layer) within minutes after the onset of the condition, which expresses itself as diffuse inner retinal whitening. ${ }^{6}$ Over the next few hours, the severity of the ischemia will worsen even further. ${ }^{6}$ Ischemia most commonly occurs in the macular region; therefore, opacification is usually limited to the posterior pole; however, in more peripheral areas, the non-perfused inner retina retains its transparency due to the lack of blood supply to the area. ${ }^{6}$ The foveola, in contrast to the macula, which contains only the photoreceptor layer that is supplied by choroidal circulation, does not show any indications of opacification or haziness. ${ }^{6}$ The traditional "Cherry Red Spot" discovery is created by a contrast between the perifoveolar whitening caused by infarction and the retinal pigment epithelium under the foveola, which is visible under the microscope. ${ }^{11}$ Remember that the cilioretinal artery, which is a branch of the choroidal arterial supply, is present in $15 \%$ to $30 \%$ of the general population. A portion of the retina surrounding the macula, which is the portion of the retina responsible for central vision, is supplied by this blood vessel. It is possible for patients who have a cilioretinal artery to retain some degree of central vision after having surgery to repair it since this area of the retina is still being perfused at the time of surgery to repair it. ${ }^{12}$ When a cilioretinal artery becomes clogged, the visual prognosis is dictated by how much of the macular blood supply is dependent on this vessel, regardless of the reason.

Where in the body the blockage of CRA takes place is not known at this point. When it comes to embolic occlusive illness, the narrowest section of the central retinal artery, which is positioned at the point where it pierces the dural sheath of the optic nerve, ${ }^{13}$ is a region that is commonly implicated.

\section{Risk Factors, Patient Characteristics, and the Etiology of CRAO}

It is possible to develop CRAO at any age, however it is most frequent in those over the age of sixty. Men are more impacted than women in this situation (by a factor of twothirds to one-third). The incidence of occurrence is 115 per 100,000 people in the population. ${ }^{14}$ According to current recommendations, individuals who suffer from CRAO should be treated as if they were suffering from a stroke of the eye and should be taken to the hospital for close monitoring and testing, in addition to risk factor reduction. 
The majority of instances of CRAO are caused by emboli that originate in the heart, carotid arteries, or aortic arch, according to the American Heart Association (referred to as "non-arteritic" embolisms). Please keep in mind that there are various different forms of emboli to be aware of. It is likely that any specific medical or mechanical attempts to dislodge or dissolve cholesterol emboli, platelet emboli, or red cell emboli would have a different approach. It's possible that heparin will have little effect on a cholesterol thrombus, for example. The sort of embolus that is present would decide whether or not the treatment would be effective. Some have claimed that this distribution does not necessarily apply to central retinal artery emboli, which are not apparent on fundoscopic inspection. ${ }^{15}$ The vast majority of retinal artery emboli, according to current thinking, are formed of cholesterol, with lesser proportions composed of fibrin or calcific material. $^{16}$

Having the central retinal artery completely occluded can result in retinal ischemia and infarction, in addition to temporary visual loss. Emboli are formed by carotid artery or cardiac plaque, the latter of which is typically induced by atrial fibrillation, in a manner similar to that of an ischemic cerebrovascular accident in that they are commonly caused by a carotid artery or cardiac plaque. ${ }^{17}$

Retinal ischemia -CRAO- is considered to be the ocular counterpart of a stroke of the cerebral vasculature, and patients suffering from CRAO should be treated with the utmost care. When a patient has CRAO, they are at a greater risk of developing concurrent or subsequent ischemia in other end organs, particularly in the brain. ${ }^{18}$ In spite of the fact that most ophthalmologists are aware of the systemic and neurologic implications of CRAO, the strategy to treating this condition continues to be a source of substantial ambiguity in the field. A narrow time window for effective treatment of CRAO and a high rate of serious comorbid illness exist. Thus, when CRAO is diagnosed in an ophthalmology, optometry, neurology, or primary clinic, immediate triage to an emergency department is necessary and should not be delayed to obtain further outpatient evaluation or institute other treatments. ${ }^{19}$ Stroke centers should develop relationships with community ophthalmologists and optometrists to promote efficient pathways for transfer of patients with CRAO. The vast majority of experts agree that it is vital to address the CRAO as an embolic event and to do an interdisciplinary full cardiological and neurological diagnostic workup to identify and treat potential hazards and causal factors as soon as possible after the CRAO has occurred. It is estimated that the chance of getting a stroke more than doubles within three years of having a CRAO. ${ }^{20}$ On multiple occasions, it has been demonstrated that obstruction of the central retinal artery is related with various vascular events such as myocardial infarction and acute ischemic stroke, among others. ${ }^{21}$ As a result, the American Heart Association $^{22}$ and the National Stroke Association ${ }^{23}$ recommend that all patients with retinal ischemia CRAO- should be evaluated for cardiovascular disease in the same way that other patients who have suffered a cerebral vascular accident should be evaluated for cardiovascular disease. The key risk factors for retinal artery occlusions have been discovered in numerous investigations, with nicotine usage, hypercholesterolemia, arterial hypertension, and stenosis of the internal carotid artery being the most prevalent. In individuals with hypertensive arteriosclerosis, the occurrence of CRAO is more likely, but it can also occur in patients with endocarditis on rare occasions. ${ }^{24-26}$

According to recent study, having an ipsilateral carotid artery stenosis is one of the most significant risk factors for central retinal artery occlusion. Acute imaging of the larger neck vasculature is therefore essential for appropriate therapy of this disease. ${ }^{24}$ However, despite the welldocumented link between CRAO and stroke, it is necessary to conduct a rapid evaluation of the carotid arteries in order to speed decisive therapy, particularly given the fact that ipsilateral carotid artery disease is known to occur in $50 \%$ of patients. ${ }^{27}$ The risk factors for cerebral stroke are comparable to those for heart attack and include, among other things, hypertension, diabetes mellitus, carotid artery disease, coronary artery disease, a history of transient ischemic attack or cerebral vascular accident, and smoking. ${ }^{28}$ As a matter of fact, atherosclerotic disease is the most common cause of CRAO in patients between the ages of 40 and 60, particularly in women. ${ }^{29}$ In addition, there have been other case series of CRAO that have occurred during and after angiography and stenting that have been documented. ${ }^{30}$ Patients typically report with debilitating monocular vision loss, and doctors should concentrate their efforts on doing thorough ophthalmic and neurologic exams on these patients. In some cases, such as this one, it may be difficult to see the classic "cattle trucking" or "box-carring," which is a discontinuous appearance of the vessels caused by segmentation of the blood column in the arteries, as well as 
the cherry-red spot in the macula without a magnified fundoscopic examination. ${ }^{30}$

The arterial vasculature of hypertension (HTN) patients becomes chronically restricted as a result of the cardiovascular atheromatous process caused by the disease. This results in heart failure (and as proved in the eye by the HTN retinopathy). It is possible that the chronically restricted vasculature will behave in a manner similar to that observed in the damaged tissues in the models outlined above. When HBO is introduced, these excessively constricted vessels do not contract any more, in contrast to the usual vasoconstriction response, because oxygen reaches them in less quantity and because they are already nearly totally constricted in the first place. Therefore, the elevated oxygen concentration has a full impact, and the ischemic retinal tissue receives a considerably bigger amount of extra oxygen in HTN patients than in nonHTN patients as a result of the increased oxygen concentration. $^{31}$

Complete blood count (CBC), erythrocyte sedimentation rate (ESR), C-reactive protein (CRP), prothrombin time (PT), partial thromboplastin time (PTT), INR if taking coumadin, and electrocardiogram (ECG) according to the etiology of CRAO are all recommended as part of the initial evaluation. The use of carotid ultrasonography, magnetic resonance imaging (MRI), and an echocardiography are all possible procedures for this condition. These studies are not regarded urgent for these individuals, in the same way that they are not considered urgent for stroke patients, but rather are part of a full evaluation for risk factor adjustment.

One of the most unusual disorders known to science is Susac syndrome, which is characterized by microangiopathy of the brain, retina, and middle ear. However, the actual source of the illness is still unknown at this time. There have been several reports of comparable retinal artery occlusions in the literature throughout the course of the years. Microangiopathy is thought to be caused by a combination of events including vasculitis and vasospasm, as well as immunologic components. Severe visual impairment is a risk for patients in this circumstance, and treatment choices are restricted in this situation. Patients who had acute treatment with hyperbaric oxygen within 24 hours of experiencing vision loss are believed to have retained foveal function and had their visual acuity improved to approximately $20 / 20$ in each eye. With the showing of hyperbaric oxygen-related improvements in visual acuity and improved visual field in both eyes on two consecutive occasions, the door has been opened to a novel therapy option for patients suffering from this ailment. $^{32}$

According to one research, retinal artery occlusion caused by CaHA filler injection is a rare but potentially life-threatening condition. In one case, HBOT was used as an adjuvant treatment, and the patient's vision and retinal circulation improved significantly. ${ }^{33}$ They came to the conclusion that HBOT appeared to be useful in the treatment of iatrogenic retinal artery occlusion within the first 24 hours of administration. Generally speaking, it is believed that a retrograde embolic mechanism is responsible for the pathogenesis of RAO after cosmetic facial filler injections are performed. ${ }^{34}$ Whenever the injection pressure is greater than or equal to the systolic arterial pressure, the injected material displaces arterial blood and travels proximally past the site of the retinal artery's origin, causing it to become blocked. By pressing the plunger, the ensuing column of material is driven into the ophthalmic artery and its branches, where it is then depressed by the arterial systolic pressure, which is then depressed as well. ${ }^{35}$

\section{CRAO in Young Population}

CRAO is a condition that is most frequently observed in the elderly and is characterized by clinical symptoms that are suggestive of atheromatous emboli. It is not prevalent among people of a younger age. In this age range, there is a scarcity of information about the risk factors associated with disease. A number of factors contributed to the pathogenesis, including cardiac, valvular, and vascular inflammatory illnesses. ${ }^{36}$ In accordance with the findings of Wenzler et al, hyperhomocysteinemia is a risk factor. ${ }^{37}$ According to the findings of another study, it was one of the most common cause of arterial occlusive disease in young people in India. ${ }^{38}$ There are a variety of disorders that can lead to hyperhomocysteinemia, including cystathionine b-synthase insufficiency, MTHFR mutation, and deficiencies in the metabolism of folate and vitamin B12. It is crucial to explore the genetic propensity to thrombosis in children as a potential cause of arterial occlusive disease in this age group. It is possible that the MTHFR gene polymorphism will cause a reduction in the enzyme activity of the MTHFR enzyme, which will result in hyperhomocysteinemia, which will impact the vascular endothelium and may result in the development of occlusive vascular disease. ${ }^{39}$ Some researchers believe that having the C677T mutation in the MTHFR gene is a risk 
factor for hyperhomocysteinemia (high homocysteine levels). ${ }^{40}$

Rarely does congenital cardiac anomaly-related emboli manifest itself as CRAO. An unusual complication of persistent truncus arteriosus (PTA) and a single atrium has been recorded in a female patient. Young-onset CRAO is related with a number of medical disorders, such as hyperhomocysteinemia, temporal arteritis, SLE, sickle cell disease, platelet-aggregation abnormalities, and migraine headaches, among others. ${ }^{41}$ In contrast, cardiogenic emboli are a very rare cause of CRAO, accounting for only $1 \%$ of all cases. In spite of the fact that atrial fibrillation and left ventricular dysfunction are the most common cardiac causes of congestive heart failure, extracardiac factors (mainly aortic and carotid plaques) have been proven to be related with this illness in the great majority of instances. ${ }^{42}$ Unfortunately, in around $45 \%$ of the patients studied, the source of the embolism was still unknown at the time of that study. Some of the other theorized reasons include infective endocarditis, left atrial thrombosis and myxomas, aortic arch atheroma, mitral annulus calcification, left atrial appendage thrombus, valvular anomalies, papillary fibroelastoma, and patent foramen ovale. ${ }^{43}$

\section{CRAO's Natural Prognosis}

Patients with CRAO often appear with severe, painless monocular vision loss. Patients with CRAO have final visual acuity that ranges from near normal (in a minority of patients who have accessory perfusion of the macula via a cilioretinal artery) to counting fingers or worse; $93.2 \%$ of patients with non-arteritic CRAO who do not have cilioretinal artery sparing will have final visual acuity of counting fingers or worse. It is possible that some CRAO patients experience spontaneous visual recovery within a few hours of vision loss and do not seek medical attention, despite the fact that a limited degree of spontaneous visual improvement is experienced by patients, typically within the first 7 days following vision loss. Even for this kind of natural course of CRAO, an ophthalmologist should be visited as soon as possible after the onset of initial symptoms and immediate assessment including evaluation of vascular risk factors is needed. ${ }^{2}$ In the natural course of CRAO, the artery's reperfusion occurs over several weeks, and this is characterized by a slow and steady improvement in blood flow. The Snellen chart shows that only $10 \%$ of patients with spontaneous reperfusion have considerable improvement (15 ETDRS (Early
Treatment Diabetic Retinopathy Study) letters or three lines in the Snellen chart). ${ }^{44}$

\section{CRAO: A Comprehensive Treatment Approach}

It was not until 2006 that hyperbaric oxygen therapy was approved as a treatment option for patients suffering from chronic respiratory apnea. In spite of this, it remains a largely underutilized modality, in part due to a lack of availability and a lack of understanding among the ophthalmological community. ${ }^{45}$ HBOT is one of the treatment options available to CRAO patients and it showed clinical effectiveness if applied in proper time frame which could be less than 24 hours after its initial onset.

\section{HBOT Has a Specific Mechanism of Action}

The principal benefits of HBO therapy are hyperoxygenation and a reduction in the size of gas bubbles in the bloodstream. Twenty-one percent of the air we breathe is made up of oxygen, which is the most critical substrate for metabolic processes. An important characteristic of HBOT is that it involves the delivery of $100 \%$ oxygen under conditions of atmospheric pressure that are two- to threefold greater than those found in normal air at the surface of the planet. During HBOT, the arterial oxygen pressure and tissue oxygen pressure can both rise to as high as 2000 millimeters of mercury and 400 millimeters of mercury, respectively. An increase in gradient (or the transfer of oxygen into tissues) by a factor of twenty as a result of the application of oxygen at this pressure can have a range of favorable impacts on biochemical, cellular, and physiological processes, among other things. ${ }^{46}$ When it comes to the healing process, oxidants have the capacity to operate as cellular messengers. ${ }^{47}$ A higher concentration of oxygen in the bloodstream allows tissues to heal themselves more effectively. When done in a controlled atmosphere, normal breathing in room air can result in an oxygen hemoglobin saturation in the blood of nearly $100 \%$. Because of the high atmospheric pressure required to inhale $100 \%$ oxygen, a rise in the solubility of oxygen in the blood occurs, which is helpful (from 0.3 up to 6 vol percent). ${ }^{48}$ This amount of plasma-dissolved oxygen, despite the fact that it is tiny, is sufficient to supply the oxygen requirements of the body's tissues. ${ }^{48}$ It has been established that when the partial pressure of oxygen is elevated in the ocular tissues, such as the vitreous body 
and inner retina, the choroidal vasculature is capable of supplying an increased amount of oxygen to the ocular tissues. ${ }^{49}$ It is acceptable to administer hyperbaric oxygen through medical compression chambers in the presence of a retinal vascular obstruction to preserve tissue life until reperfusion can be achieved. ${ }^{50}$

\section{The Rationale for HBOT in CRAO}

HBOT has been shown to be effective in the treatment of ocular vascular disorders, according to some reports. ${ }^{51}$ The interaction between free oxygen radicals and nitric oxide, which occurs in conjunction with the autoregulation that occurs during this treatment, is most likely the cause of the constriction of the retinal arteries. In the first 10 minutes after initiating HBOT, there is a considerable reduction in blood flow. Rapid vasodilatation occurs rapidly after the completion of hyperbaric oxidation treatment as a result of the enhanced production of nitric oxide and the usage of free oxygen immediately following the end of the treatment. ${ }^{52}$ While HBOT causes constriction of the retinal arteries, oxygen saturation increases to up to $23 \%$, and the retina is not harmed as a result. ${ }^{53}$ To be more specific, the improvement in retinal and macular oxygenation that occurs as a result of hyperoxia-dependent improvements in oxygenation, particularly in areas with deficient perfusion and interstitial edema or a thickened basal membrane, as well as vasoconstriction, which prevents fluid leakage that leads to retinopathy, is responsible for the beneficial effect of HBOT in treating retinal vascular diseases. ${ }^{51}$ It is still debated whether or not HBO is effective in treating retinal vascular occlusions associated with macular edema, even though current clinical evidence supports its use in the treatment of such occlusions. Concerns have been raised about the production of excess free radicals and the placebo effect. The choroidal circulation produces $60 \%$ of the oxygen required for retinal function under normal conditions, and this number rises up to $100 \%$ when the patient is exposed to hyperbaric conditions. ${ }^{54}$ If you use $\mathrm{HBO}$ to treat CRAO, the potential advantage is that the increased blood flow from the collateral and choroidal circulations can cover the metabolic demands of retinal cells, while the central retinal artery re-cannulates naturally, allowing you to keep your retinal tissue. This notion, on the other hand, is not supported by any evidence. As previously mentioned, hyperbaric oxygen has been proven to have the capacity to attenuate both tissue edema and ischemia-reperfusion harm during recanalization. The oxygen levels in the choroidal circulation must be adequately high in order for the inner retinal layers to continue to function. It is possible that enough oxygen will diffuse downstream to allow them to sustain some degree of viability if this is accomplished. As evidenced by the use of CRAO animal models, researchers have demonstrated that normal inner retinal layer architecture can be preserved when choroidal oxygen levels are elevated enough to allow for diffusion into the inner retinal layers. In order to test this concept, CRAO animal models have been deployed. ${ }^{55}$ In animal models, it has been established that collateral circulation is capable of supplying the retina with $100 \%$ of the oxygen it needed in order to function properly under hyperbaric conditions. Furthermore, in an animal experiment utilizing a CRAO mice model, Gaydar et al found in a study that HBOT reduced cell loss from $58 \%$ to $30 \%$, which was associated with enhanced survival of cells in the inner layer of the retina. ${ }^{56}$ In all animal models it should be always kept in mind that they are not the perfect model to study the use of HBOT in human CRAO and the collateralization in mice is very different from human retinal vasculature. In some cases, the use of HBOT, for example, may allow the retina's oxygen requirements to be met by the peripheral collateral circulation while the central retinal artery vessel re-cannulates. In spite of the fact that it is not known for certain, it is speculated that under hyperoxic conditions, such as those caused by hyperbaric oxygen treatment, the choroid is capable of providing the retina with $100 \%$ of the oxygen it requires, even in the presence of retinal artery non-perfusion. ${ }^{57}$

A hyperbaric therapy at $203 \mathrm{kPa}$ results in an inspired partial pressure of oxygen that is nearly 10 times larger than the partial pressure of oxygen encountered when breathing air at normal atmospheric pressure. According to this idea, higher-pressure oxygen is supposed to diffuse from the choroidal circulation or from other patent retinal arteries to reach the ischemic retina through the choroidal circulation. This reactivates cellular metabolism and maintains the retina alive, providing emboli enough time to dissolve or move on to a new site before disintegrating or moving on. In accordance with anecdotal comments from this patient cohort, the vast majority of them reported visual return after their first HBOT. This could be explained by decreasing edema in the retina, which allowed for increased visual acuity during the procedure. Moreover, data shows that HBOT would only provide a few extra hours of time for circulation to be restored to the retina at the most. The procedure will not be effective 
in cases where the retina has already been infarcted, and as a result, it is only useful in patients who appear within a short amount of time after an artery has been blocked. In light of the fact that our patients who present with CRAO within 10 hours of treatment tend to have a higher rate of improvement in vision, it would appear reasonable to administer HBOT to any patient who presents with CRAO within this time frame.

It has been postulated that the saturation of the vitreous body with oxygen is the basic reason for the use of intraocular hyperbaric oxygen therapy in ischemic ophthalmic disorders. The fact that it is a "liquid" medium does not affect the reality that it generally includes roughly $80 \%$ dissolved nitrogen until saturation, after which the nitrogen is exchanged for oxygen during the HBOT method. Because of the lower partial pressure gradient outside of the pressure chamber, oxygen desaturation of the vitreous towards the retina occurs much more slowly than saturation; as a result, it was hypothesized that the vitreous acts as an oxygen depot during the first two days of treatment. A fast rate of blood flow and a very low arteriovenous oxygen difference are observed in the choroidal circulation. Variations in oxygen tension have no substantial effect on choroid blood flow, at least when compared to changes in retinal blood flow. When decreased hemoglobin is supersaturated, oxygen pressure can grow unabated to exceedingly high levels as a result of rising amounts of free dissolved oxygen, which can result in death. Using this method, it is feasible to achieve a higher gradient of diffusion from choroidal circulation to the inner retinal layers. ${ }^{58}$

\section{HBOT's Molecular Mechanisms of Action}

The use of hyperbaric oxygen may be able to lessen the degree of ischemia neuronal injury in some patients in certain circumstances. In that ischemic neuronal damage in the retina begins with a hypoxia event, just as it occurs in the central nervous system. The time span during which ischemic tissue can recover from a hypoxic event in the event that reperfusion occurs is limited, and it is important to understand this (the ischemic penumbra). ${ }^{59}$ The depletion of adenosine triphosphate and the disruption of the calcium pump occur as a result of a decrease in oxygenation during an ischemic episode in the brain. Cell death and the accumulation of harmful chemicals are caused by the influx of calcium that happens as a result of this process. Some enzymes, such as xanthine oxidase, induce free radical damage to the organism when the blood vessels are re-opened. The absence of excitatory amino acids, which are necessary for the activation of membrane channels, also results in an increase in the accumulation of calcium, water, sodium, and chloride within the cell, which leads to additional intracellular accumulation of these elements. Apart from these potential consequences, acidosis may also contribute to the damage by causing toxic injury and catalyzing the amplification of destructive mechanisms, among other things. Hyperbaric oxygenation administered through the choroid may aid in the prevention of the buildup of such compounds as well as the facilitation of their elimination. $\mathrm{pH}$ changes may be able to be rectified in some cases. Due to the fact that the retinal tissue would remain in the ischemic penumbra during the ischemic event, the acute destruction would be reduced while the reperfusion injury would be reduced. When it comes to foveal involvement, even a tiny amount of tissue preservation can be substantial.

\section{Protocol for Hyperbaric Oxygen Treatment}

It is necessary to compress two atmosphere absolute (ATA) pressure at first. According to a study by Hayreh et al al. ${ }^{5}$ it was mentioned that there is a threshold of time beyond which ischemic tissue can no longer recover from a hypoxic event, even if reperfusion occurs. The retina can only survive 90 to 100 minutes of ischemia prior to permanent damage. So at least 90 minutes of hyperbaric oxygen application is advised. It is recommended that if vision improves sufficiently at 2 ATA, the depth be maintained at this level for 90 minutes. If vision does not improve after 30 minutes at 2 ATA, compression to 2.4 ATA is conducted, and if vision does not improve at this depth, treatment at this depth for another 90 minutes is carried out until vision improves. If there is no improvement at 2.4 ATA, the compression is increased to 2.8 ATA. After completing the initial US Navy Table No. 6, as described above, the following alternatives are available: discontinuing treatment or pursuing continued normobaric oxygen therapy. The patient is re-assessed after 4 to 6 days after receiving an additional 2 treatments at 2.8 ATA for 90 minutes each, with 5-minute air breaks every 30 minutes, twice daily. Once a plateau is reached, the majority of patients will not experience any further improvement in visual acuity and can therefore be discharged from the treatment program. It is possible that some patients will take up to eight sessions before seeing an improvement in their visual acuity; however, few if any patients will respond after this point. 


\section{The Importance of Therapeutic Window in CRAO for Obtaining the Best Results from HBOT}

Among the various elements that might influence a patient's visual prognosis, it is widely known that time elapsed between the time of diagnosis and the time of therapy is believed to be the most crucial aspect to take into consideration. With CRAO, the key pathophysiological mechanism of an early HBOT is the hyperbaric oxygen delivery to the inner and outer retinal layers through the choroid, which occurs during the early stages of the disease ${ }^{60}$ However, when is the most appropriate moment to deliver treatment is up for debate. As a general rule, the shorter time that elapses between injury and treatment, the greater the likelihood of recovering an ischemic retina that is threatened but is still functional (penumbra). ${ }^{54}$ The inner retinal cells have a time limit that must be met before they are no longer able to recover from a hypoxic event, regardless of whether or not reperfusion occurs. ${ }^{57}$ In the meta-analysis of Wu et al covered 7 RCT publications, the timing of treatment from the time of onset could not be statistically assessed due to the requirement that all RCTs be conducted within a same particular time window. ${ }^{45} \mathrm{CRAO}$ was shown to be effective in five out of six patients who underwent the treatment, which took more than one and a half hours on average. Patients' visual acuity improved significantly after the procedure was completed. ${ }^{61}$ Although numerous studies have found that HBOT is useful in the treatment of CRAO, there is disagreement about how long HBOT should be administered in this circumstance. ${ }^{54}$ According to Butler et al, the best evidence points a success within the initiation of HBOT in 12 hours of the CRAO diagnosis. ${ }^{50}$ It has been found that HBOT works best when it is started within 8 hours after the commencement of vision loss, according to researchers Hertzog and colleagues as well as Beiran and colleagues ${ }^{3,31}$ HBOT should be commenced within 24 hours of the onset of symptoms, and furtherly it has been observed that results are better if it is done within 12 hours of the onset of symptoms. According to that study, majority of patients who get hyperbaric oxygen within eight hours of acquiring symptoms experience an improvement in visual acuity of three lines or better on the Snellen chart. ${ }^{63}$ Patients who are treated within 12 hours of the onset of symptoms show the greatest indication of symptomatic improvement, according to another ophthalmology literature. ${ }^{54}$ One other group of researchers came to the conclusion that individuals with CRAO whose symptoms have been present for less than 6 to 8 hours have the greatest outcomes after getting HBO therapy. ${ }^{45}$ As a result of these different studies mentioned above in this section; there is not a definite number of initiation time but all studies main conclusion is starting HBOT as soon as possible the CRAO diagnosis is made.

\section{The Total Number of HBOT Sessions}

Another measure that can be used to assess the efficacy of HBOT is the median number of sessions completed in a given time period. There was an average of 10.5 hours of therapy throughout the investigations, which is consistent with $\mathrm{Wu}$ et al findings. It was also reported in a recent meta-analysis of seven randomized controlled trials that the median number of HBOT sessions was three, according to the findings of the study (251 eyes, with the most effective treatment length over 9 hours). ${ }^{45}$ Furthermore, according to the Undersea and Hyperbaric Medical Society, the appropriate number of HBOT sessions depends on the severity and duration of a patient's symptoms, as well as the degree to which those symptoms are responding to the HBOT treatments administered. ${ }^{54}$

\section{The Duration of HBOT}

Recent research by Wu et al found that an individual-level data set from 251 patients who got oxygen therapy was collected after a meta-analysis of seven randomized controlled trials (RCTs), This meta-analysis was focused with a special emphasis on identifying the period of treatment. According to the study's findings, treatment durations of more than 9 hours boosted the therapeutic efficacy of the intervention. ${ }^{45}$ It takes more than 9 hours of treatment for a patient to get their vision entirely restored. It was demonstrated in one of the case presentations that a CRAO patient who had hyperbaric oxygen therapy for a total of 13.5 hours saw considerable improvement in his or her visual acuity after getting the treatment. ${ }^{64}$ Each HBO therapy session can run anywhere from 45 minutes to 5 hours, with the majority of sessions lasting roughly 90 minutes or more.

\section{In CRAO, There are Certain Indicators of HBOT Success}

It has been suggested by some writers that there are four significant crucial aspects that determine the success of HBOT: the time at which HBOT is began, the degree of vessel occlusion, the kind of vessel that is occluded, and the existence of an appropriate $\mathrm{PaO} 2$ of oxygen in the blood stream. It is also believed that the etiology of the occlusion, as previously 
explained, is a crucial component in the situation. ${ }^{54,65} \mathrm{~A}$ key hurdle to effective treatment is the fact that persons with CRAO are rarely treated in an acute setting and that there is no consensus on treatment or guideline-based therapy for the condition. ${ }^{53}$ Once the tissue has been irreparably injured, it is critical to begin treatment as soon as possible after the injury. The response to HBOT will vary from person to person depending on the severity and location of the blockage. It is necessary to maintain a sufficient partial pressure of oxygen in the retinal vessels for an extended period of time in order to allow the vessels to re-canalize. This normally occurs within 72 hours of the initial occurrence. It is possible to restore retinal blood flow in as little as 72 hours after undergoing recanalization surgery. However, if ischemia and hypoxia have caused cell death and necrosis in the inner retinal layers, which are normally supplied by the retinal artery, vision may not be restored following retinal artery re-canalization. Eyesight loss in people with CRAO is widespread because the inner retinal layers, which are normally serviced by the retinal circulation, are occluded, causing them to lose their vision. However, if these layers are able to acquire sufficient oxygen through diffusion through the choroidal circulation, they will be able to continue to function. ${ }^{55}$ HBOT's ability to oxygenate the ischemic inner retinal layers is dependent on the quantity of choroid perfusion that occurs under CRAO circumstances, which is measured in microliters per second. There will be no blood flow to the posterior ciliary vessels and, thus, no collateral circulation to oxygenate the inner retina until the ophthalmic artery is totally obstructed, which is not likely in the case of complete obstruction of the ophthalmic artery. ${ }^{54}$ To keep the retina alive until circulation is restored through natural recanalization, which normally occurs within 72 hours of the incident, it is vital to maintain an acceptable partial pressure of oxygen in the bloodstream. ${ }^{54}$

\section{The Importance of Fundus Findings in the Assessment of HBOT Therapeutic Response}

Hadanny et al recently reported that patients who have a cherry-red spot (CRS) macula in the fundoscopy at presentation experience a significant increase in VA improvement as well as an increased success rate with treatment when contrasted with patients who do not have the cherry-red spot (CRS) in the fundoscopy at presentation. When comparing patients with and without CRS, it was revealed that $86.0 \%$ experienced clinically meaningful visual improvement, compared to $49.4 \%$ of patients who did not have CRS when they presented. In this context, the authors demonstrated that CRS can be utilized as a marker for irreversible anoxic retinal damage in individuals who are candidates for high-dose oxygen therapy, and that this marker can be used to guide treatment. Indeed, they advise that it should be considered as the most crucial indicator of treatment success rather than the amount of time that has passed from the onset of symptoms. ${ }^{6}$

According to the most recent findings of optical coherence tomography (OCT), this opacification and swelling of the posterior pole, which was previously discovered to be sparing the fovea (CRS), is the result of an ischemia infarction of the inner retinal tissues. After being damaged by a non-reversible infarct, the inner retinal tissues experience an increase in optical density, and this causes them to cast a shadow over the lesser optical intensity of the surrounding outer retina. ${ }^{66}$

\section{Can HBOT Be Used in Conjunction with Other Therapy Techniques to Enhance Its Effectiveness?}

Different researchers have also tried various combinations of therapeutic techniques with HBOT in the treatment of acute CRAO (eg: HBOT and ocular massage, HBOT and rTPA, HBOT and hemodilution). ${ }^{62,67-69}$ Societies have not yet approved of these varied combinations of different therapy techniques. Nevertheless, thrombolysis should be discussed in light of the rapidly developing research in recent years.

Intravenous thrombolytic medications have been used empirically to treat CRAO since the 1960s, and tPA is now delivered to $5.8 \%$ of patients treated with CRAO in the United States. ${ }^{70}$ More than half of academic neurologists administer intravenous tPA to selected patients with acute CRAO based on observational data and in the lack of other efficient therapies. ${ }^{71}$ There have been no adequate randomized clinical studies of intravenous tPA to date since earlier attempts were hampered by patient enrollment difficulties. Since the release of the meta-analysis of observational studies, intravenous tPA has been reevaluated in four current cohorts with acute CRAO within 4.5 hours of onset. ${ }^{71}$ A new meta-analysis that included these recent cohorts found that therapy within 4.5 hours had a considerable effect. $^{72}$ This analysis robustly repeated previous findings and served as the foundation for several ongoing clinical trials. Three randomized trials (THEIA, REVISION, and TenCRAOS) are being done in 
Europe to compare intravenous thrombolysis to placebo in persons with CRAO who come within 4.5 hours of symptoms commencement. ${ }^{71}$ The decision to utilize intravenous tPA for CRAO rests on a detailed conversation between the treating specialist and the affected patient, which includes an acknowledgement of the limitations inherent in the literature to date, according to the authors. $^{70,71}$ There is only one case report of concurrent use of HBO and tPA for CRAO. This patient who had recently been diagnosed with CRAO was treated with IVT (rTPA) and HBOT, which resulted in a favorable outcome. With the inclusion of a good VA result, this clinical description contributes to the current body of knowledge about the use of thrombolysis in the literature and may be considered as an alternate add - on option for the acute therapy of future CRAO cases. ${ }^{69}$ As the recent literature on thrombolysis for CRAO is increasing, there is a need to investigate the clinical efficiency of thrombolytic agent plus HBOT treatment modalities with future randomized clinical trials.

It is being investigated whether HBOT and hemodilution is effective in CRAO through a controlled, non-randomized experiment. ${ }^{62}$ The researchers conducted a comparison between HBOT and hemodilution and discovered that hemodilution alone was superior. Thirty-nine patients were given both HBOT and haemodilution, and another 29 patients were given only haemodilution alone. The mean VA improvement in the combination group was three lines, whereas the mean VA improvement in the haemodilution alone group was one line in the HBOT group. However, there was no statistically significant difference between these groups at the time of discharge or at the time of followup, indicating that the study was not statistically significant.

\section{Clinical Evidences of HBOT in the Management of CRAO}

In order to boost oxygenation of ischemic retinal regions while waiting for blood flow to be restored, HBOT is utilized. Patients who got oxygen therapy had a likelihood of visual improvement that was approximately 5.61 times greater (95\% confidence interval: $3.60-8.73$ ) than those who did not receive oxygen therapy, as compared to the control group who did not receive oxygen therapy. ${ }^{45}$ Because better outcomes are expected to occur with earlier treatment initiation, patients who present within 24 hours of the onset of symptoms may be considered for HBOT, despite the fact that there is currently no consensus in the current literature regarding the time period during which HBOT may be beneficial. ${ }^{55}$

A meta-analysis conducted by Wu et $\mathrm{al}^{45}$ in this regard is particularly noteworthy because it comprised seven randomized controlled trials involving 251 individuals who were treated with some type of "oxygen therapy" for retinal artery occlusions and found that the treatment was effective. The inhalation of carbogen ( $95 \%$ oxygen and $5 \%$ carbon monoxide) (which is beyond the scope of this review) was utilized in one study, and HBO was employed in six other investigations. The vast majority of HBO research exhibited a "low risk of bias," indicating a high likelihood of statistically valid outcomes in the vast majority of cases. It was not possible to determine whether $\mathrm{HBO}$ alone has an independently beneficial effect on visual outcome in the absence of additional treatments because five out of the six HBO studies included additional treatments (anterior chamber paracentesis, ocular massage, acetazolamide, and/or hemodilution). When it came to HBOT in CRAO research, visual acuity was the primary outcome in all of them. Patients were treated as soon as 30 minutes after the onset of symptoms in some trials, while in others, they were treated as late as 5 days after the onset of symptoms in other studies (two studies treated all patients within 12 hours, another two studies treated all patients within 48 hours, and the final two studies treated patients as late as 5 days after onset of symptoms). ${ }^{45}$ Comparing the oxygen therapy group with the non-oxygen therapy group, the oxygen therapy group showed a statistically significant increase in visual acuity in patients suffering from retinal artery blockage (odds ratio: 5.61; 95\% confidence interval: 3.60-8.73). Following the outcomes of this meta-analysis, it was shown that oxygen therapy (most often HBOT) had some visual benefits when combined with other therapies.

As previously stated, treatment of CRAO with hyperbaric oxygen treatment is rated as level IIb evidence in accordance with the American Heart Association's categorization of evidence system. HBOT is considered to be acceptable but a weak recommendation, yet there is no evidence to support this assumption strongly from level I research. The Undersea and Hyperbaric Medical Society recommends that patients seeking treatment for CRAO seek hyperbaric oxygen therapy within 24 hours of being identified with the disease, according to the society. ${ }^{54}$ It is also stated that cases in which clinical improvement has been reported have been delayed by less than 12 hours have shown the strongest evidence.

As reported by the European Committee of Hyperbaric Medicine (ECHM) at the tenth European Consensus 
Conference on Hyperbaric Medicine (2016), Type 2 therapy is recommended when HBOT is recommended because it is supported by acceptable levels of evidence, and Level $\mathrm{C}$ therapy is recommended when proper randomized controlled trials - RCTs - are not possible, but there is ample and international evidence. ${ }^{72}$ Additionally, HBOT is classified as IIb (Usefulness/effectiveness is unknown/ unclear/uncertain or not well-established) according to the clinical practice guidelines of the American College of Cardiology/American Heart Association (ACC/AHA), which means that it is not unreasonable to perform a procedure or administer treatment in this situation. ${ }^{73,74}$

In support of the hypothesis that hyperbaric oxygen can enhance outcomes following CRAO, a considerable number of case series and clinical investigations have been reported up to date. Aisenbrey et al discovered that a mean improvement of 2 lines in visual acuity was observed in eight patients who received HBOT. ${ }^{75}$ More than $80 \%$ of patients in Beiran et al series reported subjective improvement in eyesight after undergoing $\mathrm{HBO}$ treatment, compared to less than $30 \%$ of patients in the control group. ${ }^{31}$ During a research involving 21 patients, 19 of them reported subjective improvement in eyesight, which was corroborated by visual acuity tests in 13 of them. ${ }^{66}$ According to Cope and colleagues, more than half of the patients who had HBOT experienced a visual acuity improvement of greater than 2 lines. The researchers pooled data from their case series of 11 patients with data from two other case series of HBO therapy, resulting in a total of 51 individuals in the combined study. After conducting an investigation into the visual acuity outcomes of 51 HBO-treated patients and 29 patients in a control group, the researchers discovered that the HBO group experienced an average improvement of 3 lines of vision, whereas patients in the control group experienced an average improvement of only 1 line of vision. This finding was supported by another research. ${ }^{61}$

According to Coelho et $\mathrm{al}^{63} 71.4 \%$ of the patients saw a clinically significant improvement in BCVA after HBOT, and Hadanny et $\mathrm{al}^{6} 67.2 \%$ of the patients experienced a clinically significant improvement in BCVA after HBOT. According to Hadanny et al, a statistically significant mean improvement in BCVA (logMar) was seen, with a decrease in BCVA from 2.14 to $1.61(0.01 \log M a r)$ in $67 \%$ of the patients and a gain in BCVA of $>0.3 \log$ Mar in the remaining patients. A further improvement from $\mathrm{HM}$ to $\mathrm{CF}$ has been regarded as clinically important, and it correlates to a four-line interval between the two states between which the improvement occurred (in 0.1 log-unit steps). ${ }^{6}$

\section{Contraindications of HBOT}

Absolute contraindications to $\mathrm{HBO}$ treatment are distinguished from relative contraindications. An untreated pneumothorax, as well as certain chemotherapy treatments, are absolute contraindications. An existing pregnancy, epilepsy, a severe claustrophobia, an acute infection (especially of the upper respiratory tract), chronic sinusitis, or an uncontrollably high fever are all relative contraindications to receiving this treatment. There are no known contraindications to pressure chamber treatment in emergency situations, according to the guidelines of the GTUM (Gesellschaft für Tauch- und berdruckmedizin), the EUBS (European Underwater and Baromedical Society), and the UHMS (Underwater and Hyperbaric Medical Society). Extremely severe or long-lasting side effects are extremely rare. When there are problems with pressure equalization, pressure differences can cause damage to closed, air-filled body cavities as well as adjacent structures, which can be dangerous (barotrauma). Patients suffering from chronic bronchitis, asthma, or pulmonary emphysema are at a higher risk of developing lung complications. It is possible that the brain will experience a transient increase in oxygen sensitivity (oxygen cramp, feeling disturbances). This is always reversible and has no negative ramifications.

\section{HBOT Safety}

HBOT has been demonstrated to be safe as long as the treatment procedures for each portion are no longer than 120 minutes in length and the oxygen pressure is less than 3 atmospheres. When performed under adequate medical supervision, the use of high-pressure oxygen treatment is generally viewed as benign, safe, and associated with minimum risk. ${ }^{76}$

It was discovered that $5.5 \%$ of the CRAO population experienced modest and temporary adverse effects, such as middle ear or sinus barotraumas, which recovered fully within a few days of being exposed to HBO. ${ }^{44,77,78}$ When a person's middle ear and the external environment are not equalized, it is known as barotrauma. This term refers to the stretching and tearing of their tympanic membrane as a result of their inability to equalize their pressure gradient, as explained above. Furthermore, HBO is performed in numerous sessions over a period of several days and necessitates the use of specialist equipment. A single 
treatment for CRAO patients with HBO cannot be called evidence-based due to the minimal number of research that have established its effectiveness.

As a result of the barotrauma effect caused by the sudden pressure change, one of the most common side effects of HBOT is oxygen convulsion. ${ }^{79}$ Other side effects include middle ear, cranial sinus, and lung rupture, which present with mild to moderate ache as a result of the sudden pressure change; and claustrophobia, which can occur in cabins with only one patient as a result of the sudden pressure change. Also likely are chest tightness, retrosternal burning sensations, and acute lung dysfunctions such as a dry cough while suffering from the condition. ${ }^{64}$ To avoid these negative consequences, seek medical advice and action as soon as possible after exposure to the HBO. HBOT, when compared to other forms of treatment, such as paracentesis, is a non-invasive, safe procedure that may be utilized for a range of purposes and has been demonstrated to be safe for patients with CRAO.

It is possible that some patients would notice a narrowing of their visual field when HBOT is delivered during the fourth hour, depending on their condition. Oxygen toxicity is characterized by eyelid twitching, which is often a warning sign that a full-blown seizure is likely to occur. ${ }^{64}$ Experiments have shown that hyperoxia can cause retinal damage at extremely high concentrations, which has been documented in the literature. Several cases have been reported, including one in which there were retinal vascular abnormalities and another in which there was a brief unilateral vision impairment. A diagnosis of multiple sclerosis had been made for both of them. ${ }^{80}$ It is not recommended to use HBOT in patients who are receiving therapeutic intraocular gas because it can cause a significant increase in intraocular pressure (IOP) in the eyes. ${ }^{81}$ Several studies have found that HBOT has no substantial effect on the eyes' ability to see clearly. ${ }^{82}$ On the other hand, according to the findings of another study, this therapeutic procedure has the potential to cause reversible myopia. ${ }^{83} \mathrm{It}$ has also been demonstrated that the use of acute hyperbaric stress in individuals undergoing corneal refractive surgery does not result in any changes in their refractive error. ${ }^{83}$ A stimulation of fibroblastic proliferation in keratorefractive surgery procedures, particularly photorefractive keratectomy operations, might result in unstable refractive effects and undesirable outcomes. Therefore, all keratorefractive operations in patients who are undergoing HBOT should be postponed unless otherwise recommended unless otherwise suggested by the physician. Even though ocular problems associated with HBO treatment are relatively rare, they can develop in some instances. Those who use HBOT for an extended period of time, particularly older adults who use the treatment on a regular basis, are at greater risk of developing the most serious consequence, nuclear cataract development, which is caused by oxidative stress in lens proteins. ${ }^{83}$ It was discovered that extended HBO therapy had a negative effect on the human lens. ${ }^{84} \mathrm{~A}$ total of 15 of their patients were wearing clear lenses in their eyes prior to the procedure. During the HBOT, seven patients $(46.6 \%)$ acquired nuclear cataracts, which was a complication of the procedure. According to the data, nuclear vacuoles were found in ten out of twenty-five patients (44\%) who got HBOT treatment. To a certain extent, it was discovered that both nuclear cataract production and the formation of nuclear vacuoles are reversible processes. ${ }^{85,86}$ As part of the research undertaken by Schaal et $\mathrm{al}^{85}$ bovine lenses were placed in an organ culture for seven days and subjected to four distinct combinations of ambient pressure and oxygen concentration during that time. As a result of their research, they came to the conclusion that high oxygen loads had a toxic effect on bovine lenses, and they went on to say that, in addition to being higher in oxygen partial pressure and greater in number of exposures, the higher the oxygen partial pressure and more exposures resulted in more severe changes in the lens. Scientists theorized that the presence of oxygen contributed to the formation of human cataracts. ${ }^{83}$ Fledelius and colleagues employed a typical hyperbaric oxygen treatment regimen, which consisted of a 95-minute session at $95 \%$ oxygen at 2.5 atmospheres delivered every day from Monday to Friday for a total of 30 sessions, with each session lasting 95 minutes. Those who received results, on the other hand, were limited to 17 individuals. All 17 patients were subjected to myopia induction during their treatment $(100 \%) .{ }^{86}$ HBOT does not appear to be associated with ocular problems when the pressure and duration of exposures are employed in clinical practice, contrary to popular belief. The development of myopia, which is the most visible issue and is also the most easily reversible, is the most easily reversible.

Using children ranging in age from two months to eighteen years, Waisman and colleagues evaluated the effectiveness and safety of high-pressure oxygen therapy. ${ }^{87}, 139$ children were enrolled in the study, and none of them experienced an ophthalmologic problem.

\section{Conclusions}

It is difficult to perform large-scale randomized controlled trials for CRAO since it happens at such a low frequency when compared to other ischemic episodes, which makes it 
challenging to find a cure. In the limited randomized controlled trials that have been published, it has been proved that no proven intervention is more effective than doing nothing. ${ }^{88}$ Prospective trials with well-designed study designs are still required in order to find the most effective treatment for CRAO. Previous studies employing HBOT as a safe alternative indicated improvement in the low visual acuity associated with CRAO prior to therapy. While waiting for the best possible outcome, ophthalmologists will continue to do everything they can to increase the oxygenation of the ischemic retina in the meanwhile. The use of HBOT may be a clinically relevant and approved therapy option for individuals who present within 24 hours of the onset of CRAO symptoms. CRAO patients who have been treated with HBOT have the advantage of having a low risk profile of treatment modality. HBOT can be employed to try to enhance the visual prognosis in this population.

\section{Disclosure}

The author reports no conflicts of interest in this work.

\section{References}

1. Leavitt JA, Larson TA, Hodge DO, Gullerud RE. The incidence of central retinal artery occlusion in Olmsted County Minnesota. Am J Ophthalmol. 2011;152(5):820-823. doi:10.1016/j.ajo.2011.05.005

2. Hayreh SS, Zimmerman MB. Central retinal artery occlusion: visual outcome. Am J Ophthalmol. 2005;140(3):376-391. doi:10.1016/j. ajo.2005.03.038

3. Hertzog LM, Meyer GW, Carson S, Strauss MB, Hart GB. Central retinal artery occlusion treated with hyperbaric oxygen. $J$ Hyperb Med. 1992;7:33-42.

4. Hayreh SS, Zimmerman MB, Kimura A, Sanon A. Central retinal artery occlusion. Retinal survival time. Exp Eye Res. 2004;78 (3):723-736. doi:10.1016/S0014-4835(03)00214-8

5. Hayreh SS, Weingeist TA. Experimental occlusion of the central artery of the retina. IV: retinal tolerance time to acute ischaemia. $\mathrm{Br}$ J Ophthalmol. 1980;64(11):818-825. doi:10.1136/bjo.64.11.818

6. Hadanny A, Maliar A, Fishlev G, et al. Reversibility of retinal ischemia due to central retinal artery occlusion by hyperbaric oxygen. Clin Ophthalmol. 2016;11:115-125. doi:10.2147/OPTH. S121307

7. Hayreh SS. The ophthalmic artery. Br J. Ophthalmol. 1962;46 (4):212. doi:10.1136/bjo.46.4.212

8. Olson EA, Lentz K. Central retinal artery occlusion: a literature review and the rationale for hyperbaric oxygen therapy. Mo Med. 2016;113(1):53-57.

9. Lee KE, Klein BE, Klein R, Meuer SM. Association of retinal vessel caliber to optic disc and cup diameters. Invest Ophthalmol Vis Sci. 2007;48(1):63-67. doi:10.1167/iovs.05-1203

10. Yu DY, Yu PK, Cringle SJ, Kang MH, Su EN. Functional and morphological characteristics of the retinal and choroidal vasculature. Prog Ret Eye Res. 2014;40:53-93.

11. Suvarna JC, Hajela SA. Cherry-red spot. J Postgrad Med. 2008;54 (1):54-57. doi:10.4103/0022-3859.39196

12. Stoffelns BM, Laspas P. Cilioretinal artery occlusion. Klin Monbl Augenheilkd. 2015;232(4):519-524. doi:10.1055/s-0034-1396327
13. Tayebi MA, Borba ML, Lawton MT, et al. Interdural course of the ophthalmic artery in the optic canal. J Neurosurg. 2019;132 (1):277-283. doi:10.3171/2018.6.JNS18856

14. Rumelt S, Dorenboim Y, Rehany U. Aggressive systematic treatment for central retinal artery occlusion. Am J Ophthalmol. 1999;128 (6):733-738. doi:10.1016/S0002-9394(99)00359-1

15. Arruga J, Sanders M. Ophthalmologic findings in 70 patients with evidence of retinal embolism. Ophthalmology. 1982;89 (12):1336-1347. doi:10.1016/S0161-6420(82)34626-6

16. Aldrich EM, Lee AW, Chen CS, et al. Local intraarterial fibrinolysis administered in aliquots for the treatment of central retinal artery occlusion, the Johns Hopkins Hospital experience. Stroke. 2008;39 (6):1746-1750. doi:10.1161/STROKEAHA.107.505404

17. Gilbert AL, Choi C, Lessell S. Acute management of central retinal artery occlusion. Int Ophthalmol Clin. 2015;55(4):157-166. doi:10.1097/IIO.0000000000000087

18. Golsari A, Bittersohl D, Cheng B, et al. Silent brain infarctions and leukoaraiosis in patients with retinal ischemia: a prospective single-center observational study. Stroke. 2017;48(5):1392-1396. doi:10.1161/STROKEAHA.117.016467

19. Flaxel CJ, Adelman RA, Bailey ST, et al. Retinal and ophthalmic artery occlusions preferred practice pattern ${ }^{\circledR}$. Ophthalmology. 2020;127(2):P259-P287. doi:10.1016/j.ophtha.2019.09.028

20. Chang YS, Jan RL, Weng SF, et al. Retinal artery occlusion and the 3-year risk of stroke in Taiwan: a nationwide population-based study. $\mathrm{Am}$ J Ophthalmol. 2012;154(4):645-652. doi:10.1016/j.ajo.2012.03.046

21. Lee J, Kim SW, Lee SC, et al. Co-occurrence of acute retinal artery occlusion and acute ischemic stroke: diffusion-weighted magnetic resonance imaging study. Am $J$ Ophthalmol. 2014;157 (6):1231-1238. doi:10.1016/j.ajo.2014.01.033

22. Jauch EC, Saver JL, Adams HP Jr, et al. Guidelines for the early management of patients with acute ischemic stroke: a guideline for healthcare professionals from the American Heart Association/ American Stroke Association. Stroke. 2013;44(3):870-947.

23. Johnston SC, Albers GW, Gorelick PB, et al. National stroke association recommendations for systems of care for transient ischemic attack. Ann Neurol. 2011;69(5):872-877. doi:10.1002/ana.22332

24. Callizo J, Feltgen N, Pantenburg S, et al. Cardiovascular risk factors in central retinal artery occlusion: results of a prospective and standardized medical examination. Ophthalmology. 2015;122 (9):1881-1888. doi:10.1016/j.ophtha.2015.05.044

25. Rudkin AK, Lee AW, Chen CS. Vascular risk factors for central retinal artery occlusion. Eye (Lond). 2010;24(4):678-681. doi:10.1038/eye.2009.142

26. Hong JH, Sohn SI, Kwak J, et al. Retinal artery occlusion and associated recurrent vascular risk with underlying etiologies. PLoS One. 2017;12(6):e0177663. doi:10.1371/journal.pone.0177663

27. Zhang LY, Zhang J, Kim RK, et al. Risk of acute ischemic stroke in patients with monocular vision loss of vascular etiology. J Neuroophthalmol. 2018;38(3):328-333. doi:10.1097/WNO.00000 00000000613

28. Hayreh SS, Podhajsky PA, Zimmerman MB. Retinal artery occlusion: associated systemic and ophthalmic abnormalities. Ophthalmology. 2009;116(10):1928-1936. doi:10.1016/j.ophtha.2009.03.006

29. Georgalas I, Pagoulatos D, Koutsandrea C, et al. Sudden unilateral painless loss of vision. BMJ. 2014;349(7):g4117. doi:10.1136/bmj. g4117

30. Tomsak RL, Hanson M, Gutman FA. Carotid artery disease and central retinal artery occlusion. Cleve Clin Q. 1979;46(1):7-11. doi:10.3949/ccjm.46.1.7

31. Beiran I, Goldenberg I, Adir Y, Tamir A, Shupak A, Miller B. Early hyperbaric oxygen therapy for retinal artery occlusion. Eur J Ophthalmol. 2001;11(4):345-350. doi:10.1177/112067210101100405

32. Li HK, Dejean BJ, Tang RA. Reversal of visual loss with hyperbaric oxygen treatment in a patient with susac syndrome. Ophthalmology. 1996;103(12):2091-2098. doi:10.1016/S0161-6420(96)30384-9 
33. Hsiao SF, Huang YH. Partial vision recovery after iatrogenic retinal artery occlusion. BMC Ophthalmol. 2014;14(1):120. doi:10.1186/ 1471-2415-14-120

34. Park SW, Woo SJ, Park KH, Huh JW, Jung C, Kwon OK. Iatrogenic retinal artery occlusion caused by cosmetic facial filler injections. $\mathrm{Am}$ J Ophthalmol. 2012;154(4):653-662. doi:10.1016/j.ajo.2012.04.019

35. Lazzeri D, Agostini T, Figus M, Nardi M, Pantaloni M, Lazzeri S. Blindness following cosmetic injections of the face. Plast Reconstr Surg. 2012;129(4):995-1012. doi:10.1097/PRS.0b013e3182442363

36. Greven CM, Slusher MM, Weaver RG. Retinal arterial occlusions in young adults. Am J Ophthalmol. 1995;120(6):776-783. doi:10.1016/ S0002-9394(14)72731-X

37. Wenzler EM, Rademakers AJ, Boers GH, Cruysberg JR, Webers CA, Deutman AF. Hyperhomocysteinemia in retinal artery and retinal vein occlusion. Am J Ophthalmol. 1993;115(2):162-167. doi:10.1016/S0002-9394(14)73919-4

38. Ratra D, Dhupper M. Retinal arterial occlusions in the young: systemic associations in Indian population. Indian $J$ Ophthalmol. 2012;60(2):95-100. doi:10.4103/0301-4738.94049

39. Weger M, Stanger O, Deutschmann H, et al. The role of hyperhomocysteinemia and methylenetetrahydrofolate reductase (MTHFR) C677T mutation in patients with retinal artery occlusion. Am J Ophthalmol. 2002;134(1):57-61. doi:10.1016/S0002-9394(02)01471-X

40. de Franchis R, Mancini FP, D’Angelo A, et al. Elevated total plasma homocysteine and $677 \mathrm{C}->\mathrm{T}$ mutation of the 5,10methylenetetrahydrofolate reductase gene in thrombotic vascular disease. Am J Hum Genet. 1996;59(1):262-264.

41. Lu CW, Wang J, Zhou DD, et al. Central retinal artery occlusion associated with persistent truncus arteriosus and single atrium: a case report. BMC Ophthalmol. 2015;15(1):137. doi:10.1186/s12886-0150126-8

42. Mouradian M, Wijman CA, Tomasian D, Davidoff R, Koleini B, Babikian VL. Echocardiographic findings of patients with retinal ischemia or embolism. $J$ Neuroimaging. 2002;12(3):219-223. doi:10.1111/j.1552-6569.2002.tb00124.x

43. Kramer M, Goldenberg-Cohen N, Shapira Y, et al. Role of transesophageal echocardiography in the evaluation of patients with retinal artery occlusion. Ophthalmology. 2001;108(8):1461-1464. doi:10.1016/S0161-6420(01)00641-8

44. Soares A, Gomes NL, Mendonça L, Ferreira C. The efficacy of hyperbaric oxygen therapy in the treatment of central retinal artery occlusion. BMJ Case Rep. 2017;2017:bcr2017220113. doi:10.1136/ bcr-2017-220113

45. Wu X, Chen S, Li S, et al. Oxygen therapy in patients with retinal artery occlusion: a meta-analysis. PLoS One. 2018;13(8):e0202154. doi:10.1371/journal.pone.0202154

46. Leach RM, Rees PJ, Wilmshurst P. Hyperbaric oxygen therapy. $B M J$. 1998;317(7166):1140-1143. doi:10.1136/bmj.317.7166.1140

47. Sen CK, Khanna S, Gordillo G, et al. Oxygen, oxidants, and antioxidants in wound healing: an emerging paradigm. Ann NY Acad Sci. 2002;957(1):239-249. doi:10.1111/j.1749-6632.2002.tb02920.x

48. Piantadosi CA. Physiology of hyperbaric hyperoxia. Respir Care Clin N Am. 1999;5(1):7-19.

49. Jampol LM. Oxygen therapy and intraocular oxygenation. Trans Am Ophthalmol Soc. 1987;85:407-437.

50. Butler FK, Hagan C, Murphy-Lavoie H. Hyperbaric oxygen therapy and the eye. Undersea Hyperb Med. 2008;35(5):333-387.

51. Krott R, Heller R, Aisenbrey S, Bartz-Schmidt KU. Adjunctive hyperbaric oxygenation in macular edema of vascular origin. Undersea Hyperb Med. 2000;27(4):195-204.

52. Vucetic M, Jensen PK, Jansen EC. Diameter variations of retinal blood vessels during and after treatment with hyperbaric oxygen. $\mathrm{Br}$ J Ophthalmol. 2004;88(6):771-775. doi:10.1136/bjo.2003.018788

53. Butler FK Jr. Diving and hyperbaric ophthalmology. Surv Ophthalmol. 1995;39(5):347-366. doi:10.1016/S0039-6257(05) 80091-8
54. Murphy-Lavoie H, Butler F, Hagan C. Central retinal artery occlusion treated with oxygen: a literature review and treatment algorithm. Undersea Hyperb Med. 2012;39(5):943-953.

55. Patz A. Oxygen inhalation in retinal arterial occlusion: a preliminary report. Am J Ophthalmol. 1955;40(6):789-795. doi:10.1016/00029394(55)91107-7

56. Gaydar V, Ezrachi D, Dratviman-Storobinsky O, Hofstetter S, Avraham-Lubin BC, Goldenberg-Cohen N. Reduction of apoptosis in ischemic retinas of two mouse models using hyperbaric oxygen treatment. Invest Ophthalmol Vis Sci. 2011;52(10):7514-7522. doi:10.1167/iovs.11-7574

57. Wangsa-Wirawan ND, Linsenmeier RA. Retinal oxygen: fundamental and clinical aspects. Arch Ophthalmol. 2003;121(4):547-557. doi:10.1001/archopht.121.4.547

58. Dollery CT, Bulpitt CJ, Kohner EM. Oxygen supply to the retina from the retinal and choroidal circulations at normal and increased arterial oxygen tensions. Invest Ophthalmol Vis Sci. 1969;8 (6):588-594.

59. Mangat HS. Retinal artery occlusion. Surv Ophthalmol. 1995;40 (2):145-156. doi:10.1016/S0039-6257(95)80004-2

60. Varma DD, Cugati S, Lee AW, Chen CS. A review of central retinal artery occlusion: clinical presentation and management. Eye (Lond). 2013;27(6):688-697. doi:10.1038/eye.2013.25

61. Perkins SA, Magargal LE, Augsburger JJ, Sanborn GE. The idling retina: reversible visual loss in central retinal artery obstruction. Ann Ophthalmol. 1987;19(1):3-6.

62. Menzel-Severing J, Siekmann U, Weinberger A, Roessler G, Walter P, Mazinani B. Early hyperbaric oxygen treatment for nonarteritic central retinal artery obstruction. Am J Ophthalmol. 2012;153(3):454-459. doi:10.1016/j.ajo.2011.08.009

63. Coelho PM, Ferreira AP, Gonçalves R, et al. Hyperbaric oxygen therapy following central retinal artery occlusion-a retrospective case series analysis. Oftalmologia. 2018;42:2.

64. Lemos JA, Teixeira C, Rui C, Fernandes T. Combined central retinal artery and vein occlusion associated with factor $\mathrm{V}$ leiden mutation and treated with hyperbaric oxygen. Case Rep Ophthalmol. 2015;6 (3):462-468. doi:10.1159/000442788

65. Oguz H, Sobaci G. The use of hyperbaric oxygen therapy in ophthalmology. Surv Ophthalmol. 2008;53(2):112-120. doi:10.1016/ j.survophthal.2007.12.002

66. Chen H, Chen X, Qiu Z, et al. Quantitative analysis of retinal layers' optical intensities on 3D optical coherence tomography for central retinal artery occlusion. Sci Rep. 2015;5(1):9269. doi:10.1038/ srep09269

67. Weinberger AW, Siekmann UP, Wolf S, Rossaint R, Kirchhof B, Schrage NF. Treatment of acute Central Retinal Artery Occlusion (CRAO) by Hyperbaric Oxygenation Therapy (HBO)-Pilot study with 21 patients. Klin Monbl Augenheilkd. 2002;219(10):728-734. doi:10.1055/s-2002-35687

68. Kuppers-Tiedt L, Manaenko A, Michalski D, et al. Combined systemic thrombolysis with alteplase and early hyperbaric oxygen therapy in experimental embolic stroke in rats: relationship to functional outcome and reduction of structural damage. Acta Neurochir Suppl. 2011;111:167-172.

69. Ferreira D, Soares C, Tavares-Ferreira J, Fernandes T, Araújo R, Castro P. Acute phase treatment in central retinal artery occlusion: thrombolysis, hyperbaric oxygen therapy or both? J Thromb Thrombolysis. 2020;50 (4):984-988. doi:10.1007/s11239-020-02072-0

70. Schorr EM, Rossi KC, Stein LK, Park BL, Tuhrim S, Dhamoon MS. Characteristics and outcomes of retinal artery occlusion: nationally representative data. Stroke. 2020;51(3):800-807. doi:10.1161/ STROKEAHA.119.027034

71. Mac Grory B, Schrag M, Biousse V, et al. Management of central retinal artery occlusion: a scientific statement from the American Heart Association. Stroke. 2021;52(6):e282-e294. doi:10.1161/ STR.0000000000000366 
72. Mac Grory B, Nackenoff A, Poli S, et al. Intravenous fibrinolysis for central retinal artery occlusion: a cohort study and updated patient-level meta-analysis. Stroke. 2020;51(7):2018-2025. doi:10.1161/STROKEAHA.119.028743

73. Mathieu D, Marroni A, Kot J. Tenth European consensus conference on hyperbaric medicine: recommendations for accepted and non-accepted clinical indications and practice of hyperbaric oxygen treatment. Diving Hyperb Med. 2017;47(3):24-32. doi:10.28920/ dhm47.2.131-132

74. Gibbons RJ, Smith S, Antman E. American College of Cardiology; American Heart Association. American College of Cardiology/ American Heart Association clinical practice guidelines: part I: where do they come from? Circulation. 2003;107(23):2979-2986. doi:10.1161/01.CIR.0000063682.20730.A5

75. Aisenbrey S, Knott R, Heller R, Krauss D, Rüssler G, Heimann K. Hyperbaric oxygen therapy in retinal artery occlusion. Ophthalmologe. 2000;97(7):461-467. doi:10.1007/s003470070075

76. Cope A, Eggert JV, O'Brien E. Retinal artery occlusion: visual outcome after treatment with hyperbaric oxygen. Diving Hyperb Med. 2011;41(3):135-138.

77. Plafki C, Peters P, Almeling M, Welslau W, Busch R. Complications and side effects of hyperbaric oxygen therapy. Aviat Space Environ Med. 2000;71(2):119-124.

78. Hadanny A, Meir O, Bechor Y, Fishlev G, Bergan J, Efrati S. The safety of hyperbaric oxygen treatment - retrospective analysis in 2334 patients. Undersea Hyperb Med. 2016;43(2):113-122.

79. Hampson N, Atik D. Central nervous system oxygen toxicity during routine hyperbaric oxygen therapy. Undersea Hyperb Med. 2003;30 (2):147-153.

80. Herbstein K, Murchland JB. Retinal vascular changes after treatment with hyperbaric oxygen. Med J Aust. 1984;140(12):728-729. doi:10.5694/j.1326-5377.1984.tb108366.x
81. Jackman SV, Thompson JT. Effects of hyperbaric exposure on eyes with intraocular gas bubbles. Retina. 1995;15(2):160-166. doi:10.1097/00006982-199515020-00013

82. Evanger K, Haugen OH, Irgens A, Aanderud L, Thorsen E. Ocular refractive changes in patients receiving hyperbaric oxygen administered by oronasal mask or hood. Acta Ophthalmol Scand. 2004;82 (4):449-453. doi:10.1111/j.1395-3907.2004.00290.x

83. Palmquist BM, Philipson B, Barr PO. Nuclear cataract and myopia during hyperbaric oxygen therapy. $\mathrm{Br} J$ Ophthalmol. 1984;68 (2):113-117. doi:10.1136/bjo.68.2.113

84. Huang ET, Twa MD, Schanzlin DJ, Van Hoesen KB, Hill M, Langdorf MI. Refractive change in response to acute hyperbaric stress in refractive surgery patients. $J$ Cataract Refract Surg. 2002;28(9):1575-1580. doi:10.1016/S0886-3350(02)01362-7

85. Schaal S, Beiran I, Rubinstein I, Miller B, Dovrat A. Lenticular oxygen toxicity. Invest Ophthalmol Vis Sci. 2003;44(8):3476-3484. doi:10.1167/iovs.03-0122

86. Fledelius HC, Jansen EC, Thorn J. Refractive change during hyperbaric oxygen therapy. A clinical trial including ultrasound oculometry. Acta Ophthalmol Scand. 2002;80(2):188-190. doi:10.1034/j.1600-0420.2002.800213.x

87. Waisman D, Shupak A, Weisz G, Melamed Y. Hyperbaric oxygen therapy in the pediatric patient: the experience of the Israel Naval Medical Institute. Pediatrics. 1998;102(5):E53. doi:10.1542/ peds.102.5.e53

88. Fraser SG, Adams W. Interventions for acute non-arteritic central retinal artery occlusion. Cochrane Database Syst Rev. 2009;2009(1): CD001989.
Clinical Ophthalmology

\section{Publish your work in this journal}

Clinical Ophthalmology is an international, peer-reviewed journal covering all subspecialties within ophthalmology. Key topics include: Optometry; Visual science; Pharmacology and drug therapy in eye diseases; Basic Sciences; Primary and Secondary eye care; Patient Safety and Quality of Care Improvements. This journal is indexed on PubMed
Dovepress

Central and CAS, and is the official journal of The Society of Clinical Ophthalmology (SCO). The manuscript management system is completely online and includes a very quick and fair peer-review system, which is all easy to use. Visit http://www.dovepress.com/ testimonials.php to read real quotes from published authors. 